\title{
Balancing Bilinearly Interfering Elements
}

\author{
David Carfi*, Gianfranco Gambarelli**
}

\begin{abstract}
Many decisions in various fields of application have to take into account the joint effects of two elements that can interfere with each other. This happens, for example, in Medicine (synergic or antagonistic drugs), Agriculture (anti-cryptogamics), Public Economics (interfering economic policies), Industrial Economics (where the demand of an asset can be influenced by the supply of another asset), Zootechnics, and so on. When it is necessary to decide about the dosage of such elements, there is sometimes a primary interest for one effect rather than another; more precisely, it may be of interest that the effects of an element are in a certain proportion with respect to the effects of the other. It may also be necessary to take into account minimum quantities that must be assigned.

In Carfi, Gambarelli and Uristani (2013), a mathematical model was proposed to solve the above problem in its exact form. In this paper, we present a solution in closed form for the case in which the function of the effects is bilinear.
\end{abstract}

Keywords: bargaining problems, game theory, antagonist elements, interfering elements, optimal dosage, synergies

Mathematics Subject Classification: 91A80; 91A35; 91B26; 90B50

JEL Classification: C71, C72, C78

Revised: November 25, 2014

\section{INTRODUCTION}

Many decisions in various fields of application have to take into account the joint effects of two elements that can interfere with each other. This happens, for example, in Medicine (synergic or antagonistic drugs), Agriculture (pesticides), Public Economics (interfering economic policies), Industrial Economics (where the demand of an asset can be influenced by the supply of another asset), Zootechnics, and so on. When it is necessary to decide about the dosage of such elements, there is sometimes a primary

* Research Scholar Math Department University of California Riverside California, USA Visiting Scholar IAMIS, University of California Riverside California, USA, e-mail: davidcarfi@gmail.com

** Department of Management, Economics and Quantitative Methods, University of Bergamo, Italy, e-mail: gambarex@unibg.it, corresponding author 
interest for one effect rather than another; more precisely, it may be of interest that the effects of an element are in a certain proportion with respect to the effects of the other. It may also be necessary to take into account the minimum quantities that should be assigned.

In Carfì, Gambarelli and Uristani (2013), a mathematical model was proposed to solve the above problem in its exact form. In this paper, we present a solution in closed form for the case in which the function of the effects is bilinear.

In the next two sections, the problem will be defined in general terms. In Sections 4 and 5, the case of bilinear interference (free and truncated) will be dealt with. In the following section, an algorithm will be presented for the direct calculation of solutions. At the end, we shall provide some examples of application, and we shall indicate some open problems.

\subsection{LITERATURE REVIEW}

D. Carfì (2010, 2012a) has introduced a new analytical methodology to examine differentiable normal-form games. He and various collaborators have developed the applicative aspects of the new methodology in several directions, such as Management, Finance, Microeconomics, Macroeconomic, Green Economy, Financial Markets, Industrial Organization, Project Financing and so on - see, for instance, Carfi and Fici (2012), Carfì and Lanzafame (2013), Carfi, Magaudda and Schilirò (2010), Carfì and Musolino (2015a, 2015b, 2014a, 2014b, 2013a, 2013b, 2013c, 2012a, 2012b, 2012c, 2011a, 2011b), Carfi, Patanè and Pellegrino (2011), Carfì and Perrone (2013, 2012a, 2012b, 2011a, 2011b, 2011c), Carfì and Pintaudi (2012), Carfì and Schilirò (2014a, 2014b, 2013, 2012a,2012b, 2012c, 2012d, 2011a, 2011b, 2011c), Carfì, Musolino, Ricciardello and Schilirò (2012), Carfì, Musolino, Schilirò and Strati (2013), Carfì and Trunfio (2011), Okura and Carfì (2014).

The methodology can suggest useful solutions to a specific Game Theory problem. This analytical framework enables us to incorporate solutions designed "to share the pie fairly". The basic original definition we propose and apply for this methodology is introduced also in Carfì and Schilirò (2014a, 2014b, 2013, 2012a, 2012b, 2012c, 2012d, 2011a, 2011b, 2011c) and Carfì (2012a, 2012b, 2010, 2009a, 2009b, 2009c, $2009 \mathrm{~d}, 2009 \mathrm{e}, 2008)$. The method we use to study the payoff space of a normal-form game is devisable in Carfì and Musolino (2015a, 2015b, 2014a, 2014b, 2013a, 2013b, 2013c, 2012a, 2012b, 2012c, 2011a, 2011b), and Carfì and Schilirò (2014a, 2014b, 2013, 2012a, 2012b, 2012c, 2012d, 2011a, 2011b, 2011c). Other important applications, of the complete examination methodology, are introduced in Agreste, Carfi, and Ricciardello (2012), Arthanari, Carfì and Musolino (2015), Baglieri, Carfì, and Dagnino (2012), Carfì and Fici (2012), Carfi, Gambarelli and Uristani (2013), Carfì and Lanzafame (2013), Carfì, Patanè, and Pellegrino (2011), Carfì and Romeo (2015). A complete treatment of a normal-form game is presented and applied by Carfi (2012a, 2012b, 2010, 2009a, 2009b, 2009c, 2009e, 2008), Carfì and Musolino (2015a, 2015b, 2014a, 2014b, 2013a, 2013b, 2013c, 2012a, 2012b, 2012c, 2011a, 2011b), Carfì and Perrone (2013, 2012a, 2012b, 2011a, 2011b, 2011c), Carfi and Ricciardello (2013a, 2013b, 2012a, 2012b, 2010, 2009) and Carfì and Schilirò (2014a, 2014b, 2013, 2012a,2012b, 2012c, 
2012d, 2011a, 2011b, 2011c). Carfì (2008) proposes a general definition and explains the basic properties of Pareto boundary, which constitutes a fundamental element of the complete analysis of a normal-form game.

\section{DEFINITIONS}

Let $N=\{1,2\}$ be a set of labels of the considered interfering elements (i.e., drugs, commodities, and so on) and any related effects resulting from their use (e.g., curing diseases, commodity demand, and so on). From here on, if not otherwise specified, the use of the index " $i$ " will imply "for all $i \in N$ ", with an analogous use of the index " $j$ ".

\subsection{THE QUANTITIES}

We denote the non-negative quantities of the $i$-th element as follows:

- $Q_{i}$ is the quantity effectively used;

- $Q_{i}{ }^{\max }$ is the optimal quantity if the $i$-th element is used alone;

- $Q_{i}{ }^{\text {min }}$ is the minimum necessary quantity if the $i$-th element is used alone;

- $q_{i}$ and $q_{i}{ }^{\text {min }}$ are the corresponding ratios with respect to $Q_{i}$ max:

- $q_{i}=Q_{i} / Q_{i}^{\max }$

- $q_{i}^{\min }=Q_{i}{ }^{\min } / Q_{i}{ }^{\max }$.

We call $Q, Q^{\max }, Q^{\min }, q$, and $q^{\text {min }}$ the corresponding $n$-vectors.

It is assumed that $Q_{i}{ }^{\min }<Q_{i}{ }^{\text {max }}$ and $Q_{i}{ }^{\text {min }} \leq Q_{i} \leq Q_{i}{ }^{\text {max }}$. Given such conditions, $q_{i}$ and $q_{i}{ }^{\text {min }}$ belong to the interval $[0,1]$.

\subsection{THE EFFECTS}

Let $e_{i}(q)$ be a non-negative function expressing the level of the $i$-th effect when percent quantities $q$ are used. The space of the effects is the set of points $x=\left(x_{1}, \ldots, x_{n}\right)=e(q)$ according to variations of $q$. This function should satisfy the conditions that follow.

If no elements are used, then all of the effects are null. If a single element is employed in the optimal dose for use alone, then the level of the relative effect is 1 , while the level of the effect for the other is null. Finally, if both elements are employed in the optimal doses for use alone, the resulting effects are given by vector $\delta=\left(\delta_{1}, \delta_{2}\right)$ with real positive components. In formulae:

- if $q_{1}=q_{2}=0$, then $e_{1}=e_{2}=0$;

- if $q_{1}=0$ and $q_{2}=1$, then $e_{1}=0$ and $e_{2}=1$;

- if $q_{1}=1$ and $q_{2}=0$, then $e_{1}=1$ and $e_{2}=0$;

- if $q_{1}=q_{2}=1$, then $e_{1}=\delta_{1}$ and $e_{2}=\delta_{2}$.

See Figure 1 as an example of an effect's function.

Without loss of generality, we may place the elements in order so that:

$$
\delta 1 \leq \delta 2 .
$$



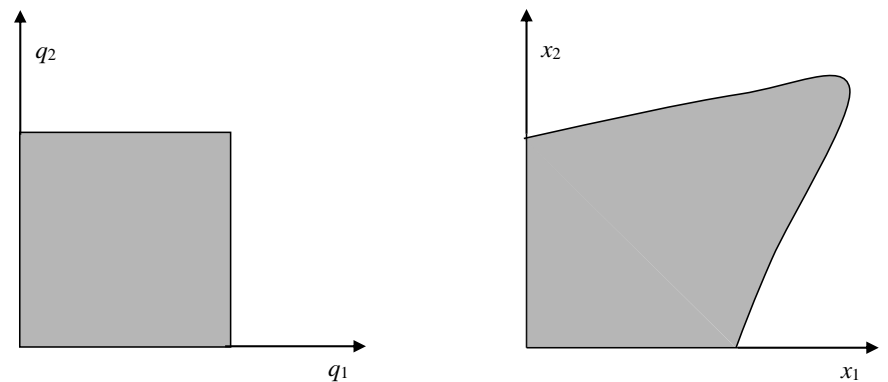

Fig. 1. Strategy space and payoff space of the game, for $n=2$

The effectfunction can be defined directly, according to the faced problem, or can be constructed on the basis of the study cases, using statistical methods and applying suitable adjustments of scale, in order to respect all of the above conditions. In this paper, we study the case in which this function is bilinear: free (Section 4) or truncated (Section 5).

\subsection{QUANTITIES AND MINIMUM EFFECTS}

We use $e_{i}^{\min }$ to indicate the minimum necessary level of the $i$-th effect. This level is derived from the function $e_{i}(q)$ given $q_{i}=q_{i}^{\min }$ and $q_{j}=0$ for the other component $j \neq i$. We use $e^{\text {min }}$ to indicate the related 2 -dimensional vector.

We assume the minimum necessary level of the $i$-th effect should not exceed 1 (if $\left.\delta_{i} \leq 1\right)$ or $\delta_{i}$ (elsewhere). Thus:

$$
e_{i}^{\min } \leq \max \left\{1, \delta_{i}\right\}
$$

\subsection{THE REQUIRED OPTIMAL RATIOS}

We use $r$ to indicate the required optimal ratio between the effects $e_{1}$ and $e_{2}$. We call $R$ the half-line centered on the origin, the inclination of which is $r$. For each point $x$ of the feasible set, we use $E$ to indicate the half-line centered on the origin, passing through $x$.

\subsection{THE FEASIBLE PARETO OPTIMAL BOUNDARY}

We shall call each point $x$ of the codomain of $e$ which is not jointly improvable a Pareto optimal effect, in the sense that if we move from that point in this set to improve the $i$-th effect, then the other effect necessarily decreases. It is easy to prove that, even here, every Pareto optimal point is a boundary point of the set of effects; we shall, therefore, call the set of Pareto optimal effects the Pareto optimal boundary.

The term feasible Pareto optimal boundary $P$ is given to the set of the points of the Pareto optimal boundary respecting the conditions $x_{i} \geq e_{i}^{\min }$ for all $i \in N$. 


\section{THE OPTIMIZATION PROBLEM}

\subsection{THE DATA}

The input data of the model is $\delta, e^{\mathrm{min}}, r$ and the option on the type of bilinear function (free or truncated).

In some applications, we do not directly know the minimal effect $e_{i}{ }^{\text {min }}$ for some element $i$, while we know the necessary minimal and optimal quantities $Q_{i}{ }^{\text {min }}$ and $Q_{i}{ }^{\max }$. It is thus possible to deduce $q_{i}{ }^{\text {min }}$, which, introduced into the equation $e_{i}(q)$, gives $e_{i}{ }^{\min }$ (as indicated in Section 2.3).

\subsection{THE OBJECTIVE}

The problem is to find the set of quantity-vectors $q^{*}$ such that the corresponding effect vectors $e\left(q^{*}\right)$ belong to the feasible Pareto optimal boundary and are such that the half-lines that join them to the origin form a minimum angle with $R$.

\subsection{EXISTENCE AND UNIQUENESS}

If the necessary minimum effects are excessive as a whole, the feasible set is empty; therefore, the problem is without solution. However, for those cases where determining the minimum quantities is open to variations, we have introduced certain indications as to modifications to be used each time. Solution uniqueness is not guaranteed in general, but the various different solutions produce the same effects (payoffs).

\subsection{SOLUTION METHODS}

Determining the optimal combination of $q$ depends clearly on the form of the effects function $e(q)$. Below, we shall present the solutions for free bilinear functions (Section 4) and for truncated bilinear functions (Section 5) providing closed form formulae and geometrical descriptions. For what concerns cases in which the effect functions are of different types, we refer to Carfì et al. (2013).

\section{FREE BILINEAR CASE}

In such cases, the function $e(q)$ of each effect is defined as follows:

$$
\begin{aligned}
& e_{1}=q_{1}\left(1-q_{2}\right)+q_{1} q_{2} q \delta_{1} \\
& e_{2}=\left(1-q_{1}\right) q_{2}+q_{1} q_{2} \delta_{2}
\end{aligned}
$$

The problem of minimizing the angle between $R$ and $E$ is defined as:

$$
\min _{q_{1}, q_{2}}\left|\frac{e_{2}}{e_{1}}-r\right|
$$

We shall examine the various types of interference separately, varying the values of $\delta$ under the constraint (1). 
We shall represent such types as graphs with corresponding numbers. In each of these graphs, the grey portion indicates the area in which $\delta$ can vary, while the bold line indicates the feasible Pareto optimal boundary.

We shall then give the solutions along with the relative steps for achieving them in the corresponding tables.

\subsection{TYPE 1 (INDEPENDENT OR SYNERGIC ELEMENTS)}

This type can be either $\delta_{1}=\delta_{2}=1$ (independent elements) or $\delta_{1}>1, \delta_{2} \geq 1$ (synergic elements) and is illustrated in Figure 2.

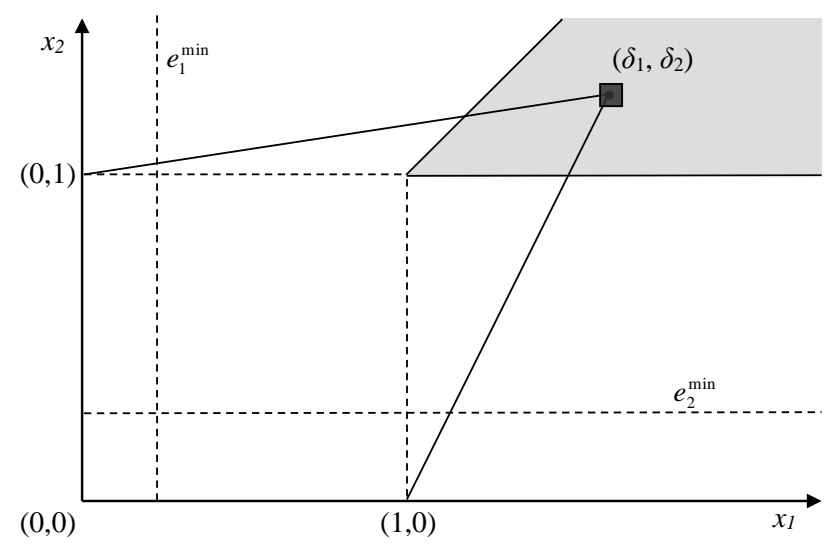

Fig. 2. $n=2$, case 1 (independent or synergic elements)

The set of effects is represented by the quadrangle having vertices $(0,0),(0,1)$, $(1,0)$, and $\left(\delta_{1}, \delta_{2}\right)$. The feasible Pareto optimal boundary is made up of the single point $\delta$. The input condition (2) guarantees the existence of the solution, given in Table 1 .

Table 1. The optimal solution in type 1

\begin{tabular}{|l|c|}
\cline { 2 - 2 } \multicolumn{1}{c|}{} & \multicolumn{1}{c|}{ values } \\
\hline optimal effects & $x^{*}=\left(\delta_{1}, \delta_{2}\right)$ \\
\hline optimal quantities & $q_{1}=1, q_{2}=1$ \\
\hline
\end{tabular}

\subsection{TYPE 2 (PARTIALLY SYNERGIC} AND PARTIALLY ANTAGONISTIC ELEMENTS)

This is the case $\delta_{1}+\delta_{2}>1, \delta_{1} \geq 1, \delta_{2}<1$. It is illustrated in Figure 3 .

The set of effects is described by the quadrangle having vertices $(0,0),(0,1)$, $(1,0)$, and $\left(\delta_{1}, \delta_{2}\right)$. 


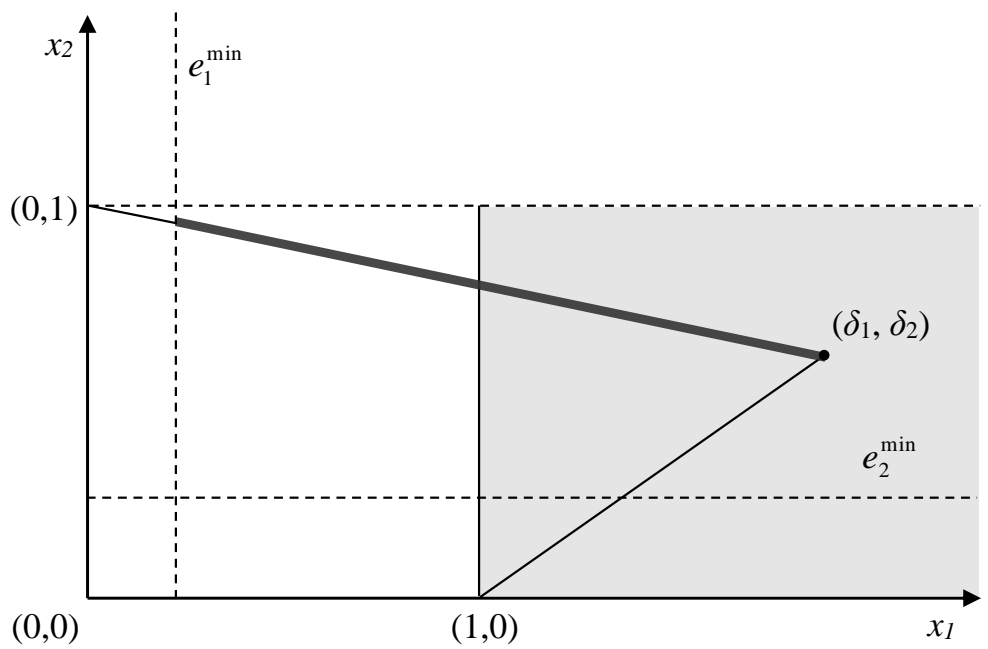

Fig. 3. $n=2$, case 2 (partially synergic and partially antagonistic elements)

In order to simplify the notations, we define:

$$
\begin{gathered}
a_{1}=\max \left(0, e_{1}^{\min }\right) \\
b_{1}=\min \left(\delta_{1}, \frac{\delta_{1}}{\delta_{2}-1}\left(e_{2}^{\min }-1\right)\right)
\end{gathered}
$$

The existence of a solution requires, besides (2), the additional condition:

$$
e_{1}^{\min } \leq b_{1}
$$

This condition results in $a_{1} \leq b_{1}$ and not-emptiness of the feasible Pareto optimal boundary. This boundary is the set of points $\left(x_{1}, x_{2}\right)$ such that

$$
\begin{aligned}
& x_{1} \in\left[a_{1}, b_{1}\right] \\
& x_{2}=\frac{\delta_{2}-1}{\delta_{1}} x_{1}+1
\end{aligned}
$$

In the event of no solution, the existence of one may be brought about by modifying $e_{1}^{\min }$ and/or $e_{2}^{\min }$ as follows:

- by fixing $e_{2}^{\min }$, we can use $e_{1}^{\min }=\frac{\delta_{1}}{\delta_{2}-1}\left(e_{2}^{\min }-1\right)$;

- by fixing $e_{1}^{\min }$, we can use $e_{2}^{\min }=\frac{\delta_{2}-1}{\delta_{1}} e_{1}^{\min }+1$.

Other ways are also open, if both $e_{1}^{\min }$ and $e_{2}^{\min }$ are modified. The solution is given in the final row of Table 2. 
Table 2. The optimal solution in type 2

\begin{tabular}{|c|c|c|}
\hline $\begin{array}{l}\text { existence } \\
\text { condition }\end{array}$ & \multicolumn{2}{|c|}{$e_{1}^{\min } \leq \min \left(\delta_{1}, \frac{\delta_{1}}{\delta_{2}-1}\left(e_{2}^{\min }-1\right)\right)$} \\
\hline $\begin{array}{l}\text { extremes of } \\
\text { the feasible } \\
\text { P.O. } \\
\text { boundary }\end{array}$ & \multicolumn{2}{|c|}{$\begin{array}{l}\mathrm{L}=\left(L_{1}, L_{2}\right)=\left(e_{1}^{\min }, \frac{\delta_{2}-1}{\delta_{1}} e_{1}^{\min }+1\right) \\
\mathrm{R}=\left(R_{1}, R_{2}\right)=\left(\frac{\delta_{1}}{\delta_{2}-1}\left(\max \left(\delta_{2}, e_{2}^{\min }\right)-1\right), \max \left(\delta_{2}, e_{2}^{\min }\right)\right)\end{array}$} \\
\hline \multirow[t]{3}{*}{$\begin{array}{l}\text { optimal } \\
\text { effects }\end{array}$} & $L_{2} / L_{1} \leq r \leq R_{2} / R_{1}$ & $\begin{array}{l}x^{*}=\left(w_{1}, w_{2}\right) \\
w_{1}=\delta_{1} /\left(r \delta_{1}-\delta_{2}+1\right) \\
w_{2}=r w_{1}\end{array}$ \\
\hline & $r>L_{2} / L_{1}$ & $x^{*}=L$ \\
\hline & $r<R_{2} / R_{1}$ & $x^{*}=R$ \\
\hline \multirow[t]{3}{*}{$\begin{array}{l}\text { optimal } \\
\text { solution }\end{array}$} & $L_{2} / L_{1} \leq r \leq R_{2} / R_{1}$ & $\begin{array}{l}q_{1}^{*}=1 /\left(r \delta_{1}-\delta_{2}+1\right) \\
q_{2}^{*}=1\end{array}$ \\
\hline & $r>L_{2} / L_{1}$ & $\begin{array}{l}q_{1}^{*}=e_{1}^{\min } / \delta_{1} \\
q_{2}^{*}=1\end{array}$ \\
\hline & $r<R_{2} / R_{1}$ & $\begin{aligned} q_{1}^{*} & =\frac{\max \left(\delta_{2}, e_{2}^{\min }\right)-1}{\delta_{2}-1} \\
q_{2}^{*} & =1\end{aligned}$ \\
\hline
\end{tabular}

\subsection{TYPE 3 (WEAKLY ANTAGONISTIC ELEMENTS)}

With this type, we have $\delta_{1}+\delta_{2} \geq 1, \delta_{1}<1, \delta_{2}<1$. This is illustrated in Figure 4 .

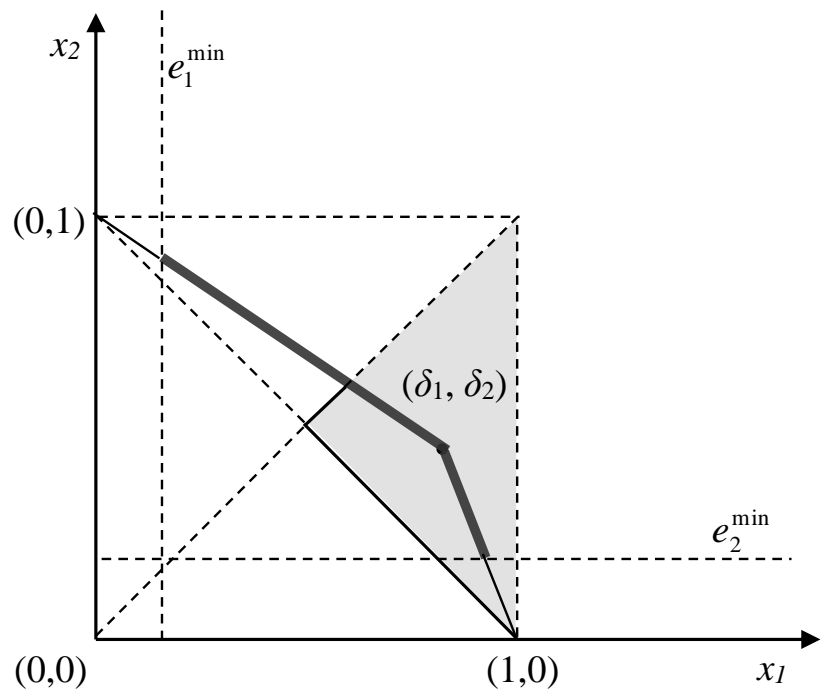

Fig. 4. $n=2$, case 3 (weakly antagonist elements) 
The set of effects is represented by the quadrangle having vertices $(0,0),(0,1)$, $(1,0)$ and $\left(\delta_{1}, \delta_{2}\right)$.

In order to simplify the notations, we define:

$$
\begin{aligned}
& a_{1}=\max \left(0, e_{1}^{\min }\right), \\
& b_{1}=\min \left(\delta_{1}, \frac{\delta_{1}}{\delta_{2}-1}\left(e_{2}^{\min }-1\right)\right) \\
& a_{2}=\max \left(\delta_{1}, e_{1}^{\min }\right), \\
& b_{2}=\min \left(1, \frac{\left(\delta_{1}-1\right)}{\delta_{2}} e_{2}^{\min }+1\right)
\end{aligned}
$$

The existence of a solution requires, besides (2), the additional condition:

$$
e_{1}^{\min } \leq \max \left(b_{1}, b_{2}\right)
$$

This condition results in $a_{1} \leq b_{1}$ e $a_{2} \leq b_{2}$ and the feasible Pareto optimal boundary is not empty. This boundary is the set of points $\left(x_{1}, x_{2}\right)$ given by $R_{1} \bigcup R_{2}$, where:

$$
R_{1}=\left\{\begin{array}{c|c}
x=\left(x_{1}, x_{2}\right) & \begin{array}{cc}
x_{2}=\frac{\left(\delta_{2}-1\right)}{\delta_{1}} x_{1}+1 \\
x_{1} \in\left[a_{1}, b_{1}\right]
\end{array} \\
\emptyset & \text { if } e_{1}^{\min } \leq \delta_{1}
\end{array}\right.
$$

and

$$
R_{2}=\left\{\begin{array}{c|c}
x=\left(x_{1}, x_{2}\right) & \begin{array}{c}
x_{2}=\frac{\delta_{2}}{\left(\delta_{1}-1\right)}\left(x_{1}-1\right) \\
x_{1} \in\left[a_{2}, b_{2}\right]
\end{array} \\
\emptyset & \text { otherwise }
\end{array} \quad \begin{array}{l}
\text { if } e_{2}^{\min } \leq \delta_{2} \\
\text { otheris }
\end{array}\right.
$$

In the event of no solution, the existence of one may be brought about by modifying $e_{1}^{\min }$ and/or $e_{2}^{\min }$ as follows:

- by fixing $e_{2}^{\min }$, we can use

$$
e_{1}^{\min }=\max \left(\frac{\delta_{1}}{\delta_{2}-1}\left(e_{2}^{\min }-1\right), \frac{\delta_{1}-1}{\delta_{2}} e_{2}^{\min }+1\right)
$$

- by fixing $e_{1}^{\min }$, we can use

$$
e_{2}^{\min }=\min \left(\frac{\delta_{2}-1}{\delta_{1}} e_{1}^{\min }+1, \frac{\delta_{2}}{\delta_{1}-1}\left(e_{1}^{\min }-1\right)\right)
$$

Other ways are also open, if both $e_{1}^{\min }$ and $e_{2}^{\min }$ are modified. The solution is given in the final row of Table 3 . 
Table 3. The optimal solution in type 3

\begin{tabular}{|c|c|c|}
\hline $\begin{array}{l}\text { existence } \\
\text { condition }\end{array}$ & $e_{1}^{\min } \leq \max (\min ($ & $\left.\left.\delta_{1}, \frac{\delta_{1}}{\left(\delta_{2}-1\right)}\left(e_{2}^{\min }-1\right)\right), \min \left(1, \frac{\left(\delta_{1}-1\right)}{\delta_{2}} e_{2}^{\min }+1\right)\right)$ \\
\hline $\begin{array}{l}\text { extremes of } \\
\text { the feasible } \\
\text { P.O. } \\
\text { boundary }\end{array}$ & $\begin{array}{l}\mathrm{L}=\left(L_{1}, L_{2}\right)=\left(\begin{array}{c}e_{1}^{\mathrm{n}} \\
+\end{array}\right. \\
\mathrm{R}=\left(R_{1}, R_{2}\right)=\left(\begin{array}{l}+\end{array}\right.\end{array}$ & $\begin{array}{l}\left(\frac{\delta_{2}-1}{\delta_{1}} e_{1}^{\min }+1\right) \times\left(e_{1}^{\min } \leq \delta_{1}\right)+ \\
\left(\frac{\delta_{2}}{\delta_{1}-1}\left(e_{1}^{\min }-1\right)\right) \times\left(e_{1}^{\min }>\delta_{1}\right) \\
\left(\frac{\delta_{1}-1}{\delta_{2}} e_{2}^{\min }+1\right) \times\left(e_{2}^{\min } \leq \delta_{2}\right)+ \\
\left.\left(\frac{\delta_{1}}{\delta_{2}-1}\left(e_{2}^{\min }-1\right)\right) \times\left(e_{2}^{\min }>\delta_{2}\right), e_{2}^{\min }\right)\end{array}$ \\
\hline \multirow{4}{*}{$\begin{array}{l}\text { optimal } \\
\text { effects }\end{array}$} & $r>L_{2} / L_{1}$ & $x^{*}=L$ \\
\hline & $r<R_{2} / R_{1}$ & $x^{*}=R$ \\
\hline & $\delta_{2} / \delta_{1} \leq r \leq L_{2} / L_{1}$ & $\begin{array}{l}x^{*}=\left(w_{1}, w_{2}\right) \\
w_{1}=\delta_{1} /\left(r \delta_{1}-\delta_{2}+1\right) \\
w_{2}=r w_{1}\end{array}$ \\
\hline & $R_{2} / R_{1} \leq r \leq \delta_{2} / \delta_{1}$ & $\begin{array}{l}x^{*}=\left(w_{1}, w_{2}\right) \\
w_{1}=-\delta_{2} /\left(r \delta_{1}-r-\delta_{2}\right) \\
w_{2}=r w_{1}\end{array}$ \\
\hline \multirow[t]{4}{*}{$\begin{array}{l}\text { optimal } \\
\text { quantities }\end{array}$} & $r>L_{2} / L_{1}$ & $\begin{array}{l}q_{1}^{*}=\frac{e_{1}^{\min }}{\delta_{1}} \chi\left(e_{1}^{\min } \leq \delta_{1}\right)+\chi\left(e_{1}^{\min }>\delta_{1}\right) q_{2}^{*}= \\
=\chi\left(e_{1}^{\min } \leq \delta_{1}\right)+\frac{e_{1}^{\min }-1}{\delta_{1}-1} \chi\left(e_{1}^{\min }>\delta_{1}\right)\end{array}$ \\
\hline & $r<R_{2} / R_{1}$ & $\begin{array}{l}q_{1}^{*}=1 \\
q_{2}^{*}=\frac{e_{2}^{\min }}{\delta_{2}} \chi\left(e_{2}^{\min } \leq \delta_{2}\right)+\frac{e_{2}^{\min }}{1-\delta_{1}} \chi\left(e_{2}^{\min }>\delta_{2}\right)\end{array}$ \\
\hline & $\delta_{2} / \delta_{1} \leq r \leq L_{2} / L_{1}$ & $\begin{aligned} q_{1}^{*} & =\frac{1}{r \delta_{1}+1-\delta_{2}} \\
q_{2}^{*} & =1\end{aligned}$ \\
\hline & $R_{2} / R_{1} \leq r \leq \delta_{2} / \delta_{1}$ & $\begin{array}{l}q_{1}^{*}=1 \\
q_{2}^{*}=-\frac{r}{r \delta_{1}-\delta_{2}-r}\end{array}$ \\
\hline
\end{tabular}

\subsection{TYPE 4 (STRONGLY ANTAGONISTIC ELEMENTS)}

This is the case $\delta_{1}+\delta_{2}<1$. This is illustrated in Figure 5 .

It may be deduced from Carfi $(2009 \mathrm{e}$, pages $42-44)$ that the set of effects is the pseudo-triangle with vertices $(0,0),(0,1)$, and $(1,0)$, delimited at North-East by the curve now to be defined. Having called $\delta_{1}^{\prime}=1-\delta_{1}$ and $\delta_{2}^{\prime}=1-\delta_{2}$, the resulting line is the union of:

- the segment of extremes $(0,1)$ and $H=\left(H_{1}, H_{2}\right)=\left(\delta_{1}^{2} / \delta_{2}^{\prime}, \delta_{1}^{\prime}\right)$,

- the segment of extremes $(1,0)$ and $K=\left(K_{1}\right.$, place $\left._{2}\right)=\left(\delta_{2}^{\prime}, \delta_{2}^{2} / \delta_{1}^{\prime}\right)$,

- the section of the curve between $H$ and $K$, having equation $x_{2}=\left(1-\sqrt{\delta_{2}^{\prime} x_{1}}\right)^{2} / \delta_{1}^{\prime}$ 


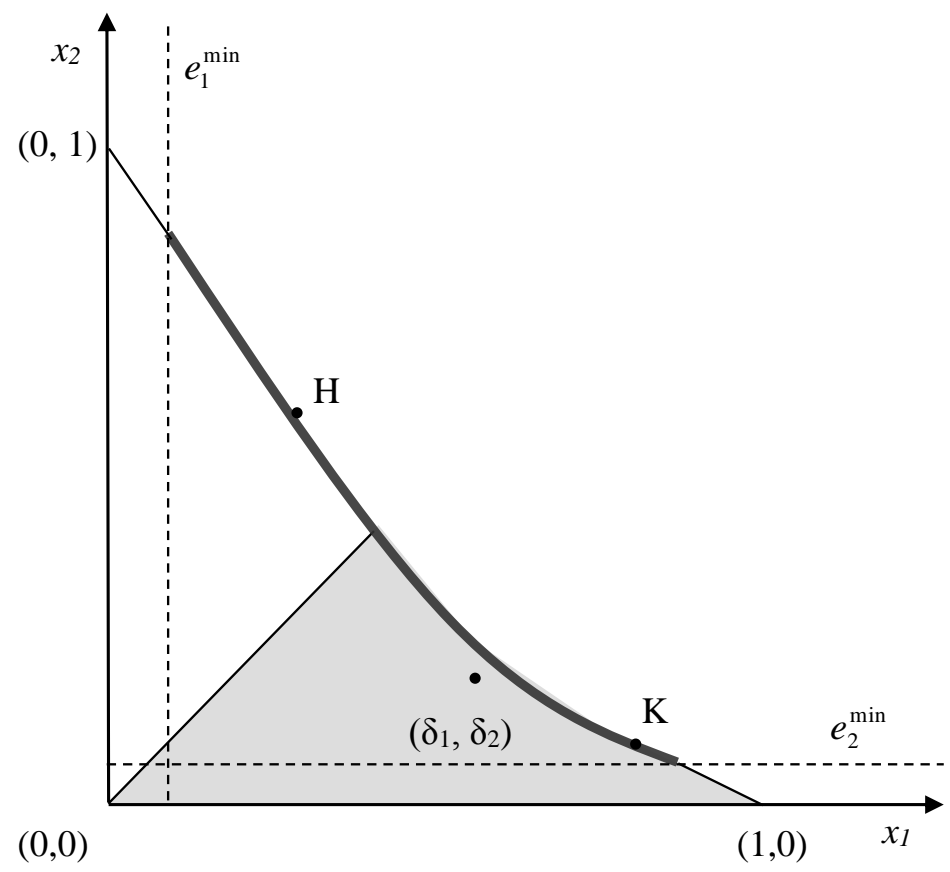

Fig. 5. $n=2$, case 4 (strongly antagonist elements)

Remark. For other examples of similar calculations, we suggest to read the papers by Carfì and Schilirò (2014a, 2014b, 2013, 2012a, 2012b, 2012c, 2012d, 2011a, 2011b, 2011c) and by Carfì (2012a, 2012b, 2010, 2009a, 2009b, 2009c, 2009d, 2009e, 2008); the interested readers could also see Carfi and Musolino (2015a, 2015b, 2014a, 2014b, 2013a, 2013b, 2013c, 2012a, 2012b, 2012c, 2011a, 2011b). Other important applications, of the complete examination methodology, are shown in Agreste, Carfì, and Ricciardello (2012), Arthanari, Carfì and Musolino (2015), Baglieri, Carfì, and Dagnino (2012), Carfi and Fici (2012), Carfì, Gambarelli and Uristani (2013), Carfì and Lanzafame (2013), Carfi, Patanè, and Pellegrino (2011), Carfì and Romeo (2015).

Note that $H$ belongs to the segment connecting $(0,1)$ and $\left(\delta_{1}, \delta_{2}\right)$, and $K$ belongs to the segment connecting $(1,0)$ and $\left(\delta_{1}, \delta_{2}\right)$; then $H_{1} \leq \delta_{1}$ and $H_{2} \leq \delta_{2}$.

In order to simplify the notations, we define:

$$
\begin{aligned}
& a_{1}=\max \left(0, e_{1}^{\min }\right), \\
& b_{1}=\min \left(H_{1}, \frac{\delta_{1}}{\left(\delta_{2}-1\right)}\left(e_{2}^{\min }-1\right)\right) \\
& a_{2}=\max \left(K_{1}, e_{1}^{\min }\right), \\
& b_{2}=\min \left(1, \frac{\left(\delta_{1}-1\right)}{\delta_{2}} e_{2}^{\min }+1\right) \\
& a_{3}=\max \left(H_{1}, e_{1}^{\min }\right), \\
& b_{3}=\min \left(K_{1}, \frac{\left(1-\sqrt{\left(1-\delta_{1}\right) e_{2}^{\min }}\right)^{2}}{1-\delta_{2}}\right)
\end{aligned}
$$


The existence of a solution requires, besides (2), the additional condition

$$
e_{1}^{\min } \leq \max \left(b_{1}, b_{2}, b_{3}\right)
$$

This condition results in $a_{1} \leq b_{1}, a_{2} \leq b_{2}$, and $a_{3} \leq b_{3}$. In this case, the feasible Pareto optimal boundary is not empty. This boundary is the set of points $\left(x_{1}, x_{2}\right)$ given by $R_{1} \bigcup R_{2} \bigcup R_{3}$, where:

$$
R_{1}=\left\{\begin{array}{c|c}
x=\left(x_{1}, x_{2}\right) & \begin{array}{ll}
x_{2}=\frac{\left(\delta_{2}-1\right)}{\delta_{1}} x_{1}+1 \\
x_{1} \in\left[a_{1}, b_{1}\right]
\end{array} \\
\emptyset & \text { if } e_{1}^{\min } \leq H_{1} \\
\text { otherwise }
\end{array}\right.
$$

and

$$
R_{2}=\left\{\begin{array}{c|c}
x=\left(x_{1}, x_{2}\right) \mid \begin{array}{ll}
x_{2}=\frac{\delta_{2}}{\left(\delta_{1}-1\right)}\left(x_{1}-1\right) \\
x_{1} \in\left[a_{2}, b_{2}\right]
\end{array} & \text { if } e_{2}^{\min } \leq K_{2} \\
\emptyset & \text { otherwise }
\end{array}\right.
$$

and

$$
R_{3}=\left\{\begin{array}{c|l}
x=\left(x_{1}, x_{2}\right) & \begin{array}{c}
x_{2}=\frac{\left(1-\sqrt{\left(1-\delta_{2}\right) x_{1}}\right)^{2}}{1-\delta_{1}} \\
x_{1} \in\left[a_{3}, b_{3}\right]
\end{array}
\end{array}\right\} \begin{aligned}
& \text { if } K_{2} \leq e_{2}^{\min } \leq H_{2} \\
& \text { and } H_{1} \leq e_{1}^{\min } \leq K_{1}
\end{aligned}
$$

In the event of no solution, the existence of one may be brought about by modifying $e_{1}^{\min }$ and/or $e_{2}^{\min }$ in a way analogous to the previous cases:

- by fixing $e_{2}^{\min }$, we can use

$$
e_{1}^{\min }=\max \left(\frac{\delta_{1}}{\delta_{2}-1}\left(e_{2}^{\min }-1\right), \frac{\left(\delta_{1}-1\right)}{\delta_{2}} e_{2}^{\min }+1, \frac{\left(1-\sqrt{\left(1-\delta_{1}\right) e_{2}^{\min }}\right)^{2}}{1-\delta_{2}}\right)
$$

- by fixing $e_{1}^{\min }$, we can use

$$
e_{2}^{\min }=\min \left(\frac{\delta_{2}-1}{\delta_{1}} e_{1}^{\min }+1, \frac{\delta_{2}}{\delta_{1}-1}\left(e_{1}^{\min }-1\right), \frac{\left(1-\sqrt{\left(1-\delta_{2}\right) e_{1}^{\min }}\right)^{2}}{1-\delta_{1}}\right)
$$

Intermediate solutions are also possible, in which both $e_{i}^{\min }$ are modified. The solution is given in the final row of Table 4 . 
Table 4. The optimal solution in type 4

\begin{tabular}{|c|c|c|}
\hline $\begin{array}{l}\text { existence } \\
\text { condition }\end{array}$ & $e_{1}^{\min } \leq \max (\operatorname{mi}$ & $\begin{array}{l}\left(H_{1}, \frac{\delta_{1}}{\left(\delta_{2}-1\right)}\left(e_{2}^{\min }-1\right)\right), \\
\left(1, \frac{\left(\delta_{1}-1\right)}{\delta_{2}} e_{2}^{\min }+1\right), \min \left(K_{1}, \frac{\left(1-\sqrt{\left(1-\delta_{1}\right) e_{2}^{\min }}\right)^{2}}{1-\delta_{2}}\right)\end{array}$ \\
\hline $\begin{array}{l}\text { extremes of } \\
\text { the feasible } \\
\text { P.O. } \\
\text { boundary }\end{array}$ & $R=\left(R_{1}, R_{2}\right)=$ & 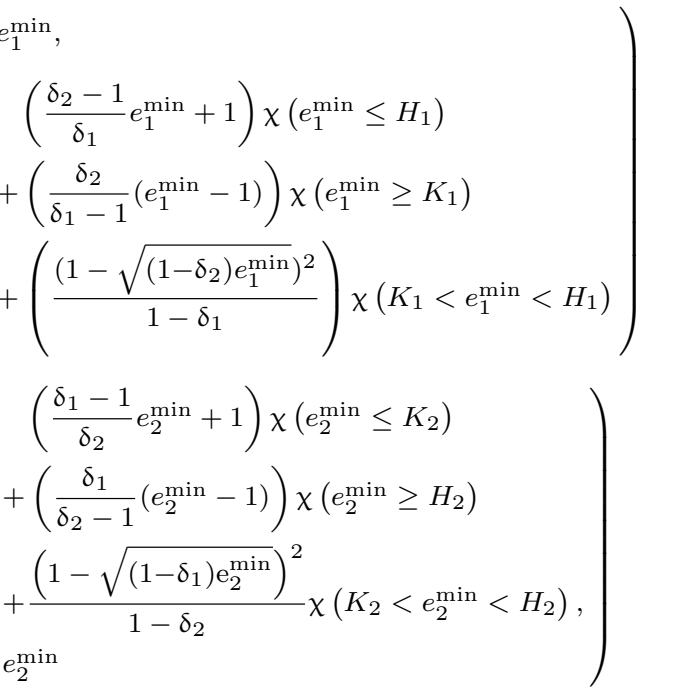 \\
\hline \multirow[t]{5}{*}{$\begin{array}{l}\text { optimal } \\
\text { effects }\end{array}$} & $r \geq L_{2} / L_{1}$ & $x^{*}=L$ \\
\hline & $r \leq R_{2} / R_{1}$ & $x^{*}=R$ \\
\hline & $\begin{array}{l}r \geq H_{2} / H_{1} \\
r<L_{2} / L_{1} \\
r>R_{2} / R_{1}\end{array}$ & $\begin{array}{l}x^{*}=\left(w_{1}, w_{2}\right) \\
w_{1}=\delta_{1} /\left(r \delta_{1}-\delta_{2}+1\right) \\
w_{2}=r w_{1}\end{array}$ \\
\hline & $\begin{array}{l}H_{2} / H_{1} \leq r \leq \\
K_{2} / K_{1} \\
r<L_{2} / L_{1} \\
r>R_{2} / R_{1}\end{array}$ & $\begin{array}{l}x^{*}=\left(w_{1}, w_{2}\right) \\
w_{1}=\left(\frac{2\left(\left(1-\delta_{2}\right)+r\left(1-\delta_{1}\right)\right)-2 \sqrt{\xi}}{2\left(\left(1-\delta_{2}\right)+r\left(1-\delta_{1}\right)\right)^{2}}\right) \\
w_{2}=r w_{1} \\
\text { where } \\
\xi=\sqrt{r\left(\delta_{1}-1\right)\left(\delta_{2}-1\right)}\end{array}$ \\
\hline & $\begin{array}{l}r \leq K_{2} / K_{1} \\
r<L_{2} / L_{1} \\
r>R_{2} / R_{1}\end{array}$ & $\begin{array}{l}x^{*}=\left(w_{1}, w_{2}\right) \\
w_{1}=\left(\frac{\delta_{2}}{\delta_{2}+r\left(1-\delta_{1}\right)}\right) \\
w_{2}=r w_{1}\end{array}$ \\
\hline $\begin{array}{l}\text { optimal } \\
\text { quantities }\end{array}$ & $r \geq L_{2} / L_{1}$ & $\begin{aligned} q_{1}^{*} & =\left(\frac{e_{1}^{\min }}{\delta_{1}}\right) \times\left(e_{1}^{\min } \leq H_{1}\right)+\chi\left(e_{1}^{\min } \geq K_{1}\right) \\
& +\left(\frac{\left(e_{1}^{\min }\left(\delta_{2}-1\right)+\eta\right.}{\eta\left(\delta_{1}-1\right)}\right) \times\left(H_{1}<e_{1}^{\min }<K_{1}\right)\end{aligned}$ \\
\hline
\end{tabular}


Table 4. cont.

\begin{tabular}{|c|c|c|}
\hline \multirow[t]{5}{*}{$\begin{array}{l}\text { optimal } \\
\text { quantities }\end{array}$} & $r \geq L_{2} / L_{1}$ & $\begin{array}{l}q_{2}^{*}=\chi\left(e_{1}^{\min } \leq H_{1}\right)+\left(\frac{e_{1}^{\min }-1}{\delta_{1}-1}\right) \times\left(e_{1}^{\min } \geq K_{1}\right) \\
\quad+\left(\frac{\eta}{1-\delta_{2}}\right) \times\left(H_{1}<e_{1}^{\min }<K_{1}\right) \\
\text { where } \\
\eta=\sqrt{e_{1}^{\min }\left(1-\delta_{2}\right)}\end{array}$ \\
\hline & $r \leq R_{2} / R_{1}$ & $\begin{aligned} & q_{1}^{*}=\chi\left(e_{2}^{\min } \leq K_{2}\right)+\left(\frac{e_{2}^{\min }-1}{\delta_{2}-1}\right) \times\left(e_{2}^{\min } \geq H_{2}\right)+ \\
&-\left(\frac{\theta+e_{2}^{\min }\left(\delta_{1}-1\right)}{\theta\left(\delta_{2}-1\right)}\right) \times\left(K_{2}<e_{2}^{\min }<H_{2}\right) \\
& q_{2}^{*}=\left(\frac{e_{2}^{\min }}{\delta_{2}}\right) \times\left(e_{2}^{\min } \leq K_{2}\right)+\chi\left(e_{2}^{\min } \geq H_{2}\right)+ \\
&+\left(\frac{\theta}{1-\delta_{1}}\right) \times\left(K_{2}<e_{2}^{\min }<H_{2}\right) \\
& \text { where } \\
& \theta=\sqrt{e_{2}^{\min }\left(1-\delta_{1}\right)}\end{aligned}$ \\
\hline & $\begin{array}{l}r \geq H_{2} / H_{1} \\
r<L_{2} / L_{1} \\
r>R_{2} / R_{1}\end{array}$ & $\begin{array}{l}q_{1}^{*}=-\frac{\delta_{1}-1}{2\left(\delta_{2}-1\right)^{2} \sqrt{\left(\delta_{1}-1\right) /\left(\delta_{2}-1\right)}} \\
q_{2}^{*}=-\frac{\delta_{2}-1}{2\left(\delta_{1}-1\right)^{2} \sqrt{\left(\delta_{2}-1\right) /\left(\delta_{1}-1\right)}}\end{array}$ \\
\hline & $\begin{array}{l}H_{2} / H_{1} \leq r \leq \\
K_{2} / K_{1} \\
r<L_{2} / L_{1} \\
r>R_{2} / R_{1}\end{array}$ & $\begin{array}{l}\text { If } \delta_{1}=\delta_{2} \\
q_{1}^{*}=-\left(\frac{1}{2} \frac{1}{\sqrt{\left(\delta_{2}-1\right) /\left(\delta_{1}-1\right)}} \frac{\delta_{2}-1}{\left(\delta_{1}-1\right)^{2}}\right) \\
q_{2}^{*}=-\left(\frac{1}{2} \frac{1}{\sqrt{\left(\delta_{1}-1\right) /\left(\delta_{2}-1\right)}} \frac{\delta_{1}-1}{\left(\delta_{2}-1\right)^{2}}\right) \\
\text { otherwise } \\
q_{1}^{*}=-\left(\frac{\delta_{1}-1+\xi}{\left(\delta_{1}-1\right)\left(\delta_{1}-\delta_{2}\right)}\right) \\
q_{2}^{*}=-\left(\frac{\delta_{2}-1+\xi}{\left(\delta_{2}-1\right)\left(\delta_{1}-\delta_{2}\right)}\right) \\
\text { where } \\
\xi=\sqrt{\left(\delta_{1}-1\right)\left(\delta_{2}-1\right)}\end{array}$ \\
\hline & $\begin{array}{l}r \leq K_{2} / K_{1} \\
r<L_{2} / L_{1} \\
r>R_{2} / R_{1}\end{array}$ & $\begin{array}{l}q_{1}^{*}=1 \\
q_{2}^{*}=-\frac{r}{r \delta_{1}-\delta_{2}-r}\end{array}$ \\
\hline
\end{tabular}

\section{TRUNCATED BILINEAR CASE}

These cases involve situations in which the effects (beyond a certain maximum level) fall to zero. The symbol $\chi$ will be used in the text to denote the indicator function; i.e.,

$$
\chi(\text { condition })= \begin{cases}1 & \text { if the condition is satisfied } \\ 0 & \text { if the condition is not satisfied }\end{cases}
$$


Using the above symbol, we can define the effect-function $e(q)$ of truncated bilinear cases as follows:

$$
\begin{aligned}
& e_{1}=\chi\left(q_{1}\left(1-q_{2}\right)+q_{1} q_{2} \delta_{1} \leq 1\right)\left[q_{1}\left(1-q_{2}\right)+q_{1} q_{2} \delta_{1}\right] \\
& e_{2}=\chi\left(q_{2}\left(1-q_{1}\right)+q_{1} q_{2} \delta_{2} \leq 1\right)\left[\left(1-q_{1}\right) q_{2}+q_{1} q_{2} \delta_{2}\right]
\end{aligned}
$$

\subsection{TYPE 1 TRUNCATED (INDEPENDENT OR SYNERGIC ELEMENTS)}

This type corresponds either to $\left(\delta_{1}=\delta_{2}=1\right)$ or $\left(\delta_{1}>1, \delta_{2} \geq 1\right)$. This is illustrated in Figure 6.

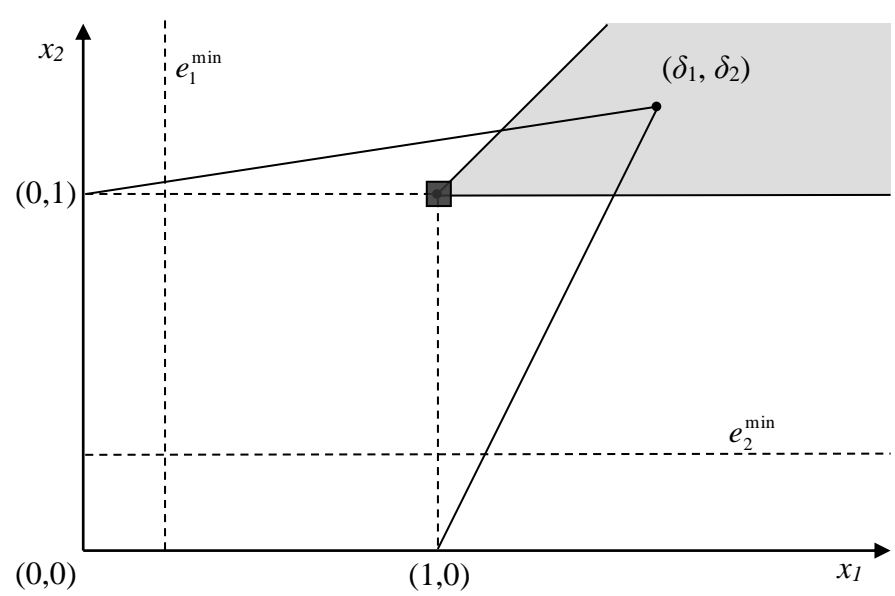

Fig. 6. $n=2$, case 1 (independent or synergic elements)

The set of effects is the quadrangle having vertices $(0,0),(0,1),(1,0)$, and $\left(\delta_{1}, \delta_{2}\right)$. The feasible Pareto optimal boundary is made up of the single point $(1,1)$. Therefore, $x_{1}=x_{2}=1$.

The input condition (2) guarantees the existence of the solution, which is given in Table 5.

Table 5. the optimal solution in type $1 T$

\begin{tabular}{|l|l|l|l|}
\cline { 2 - 4 } \multicolumn{1}{c|}{} & $\delta_{1}=\delta_{2}=1$ & $\delta_{1}>1 \delta_{2}=1$ & otherwise \\
\hline optimal effects & $x^{*}=(1,1)$ & $x^{*}=(1,1)$ & $x^{*}=(1,1)$ \\
\hline optimal & $q_{1}=\frac{1}{\delta_{1}}$ & $q_{1}=1$ & $q_{1}=\frac{1}{1+q_{2}\left(\delta_{2}-1\right)}$ \\
quantities & $q_{2}=1$ & $q_{2}=1$ & \\
& & & $q_{2}=\frac{\sqrt{\kappa^{2}-\kappa+4\left(\delta_{1}-1\right)}}{2\left(\delta_{1}-1\right)}$ \\
& & & $\kappa=\left(1-\left(\delta_{1}-1\right)+\left(\delta_{2}-1\right)\right)$ \\
\hline
\end{tabular}




\subsection{TYPE 2 TRUNCATED (PARTIALLY SYNERGIC AND PARTIALLY ANTAGONISTIC ELEMENTS)}

This is the case $\delta_{1}+\delta_{2}>1, \delta_{1} \geq 1, \delta_{2}<1$. This is illustrated in Figure 7 .

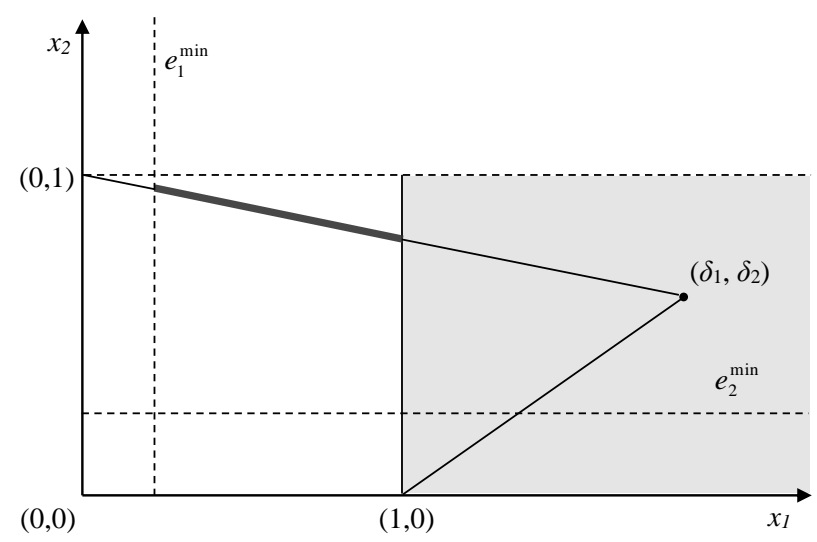

Fig. 7. $n=2$, case 2 (partially synergic and partially antagonistic elements)

The set of effects is the quadrangle having vertices $(0,0),(0,1),(1,0)$, and $\left(\delta_{1}, \delta_{2}\right)$. Although it is analogous to Type 2 in the case given in the previous paragraph, the effects cannot exceed the value of 1 in this case.

In order to simplify the notation, we define:

$$
\begin{aligned}
& a_{1}=\max \left(0, e_{1}^{\min }\right) \\
& b_{1}=\min \left(1, \frac{\delta_{1}}{\delta_{2}-1}\left(e_{2}^{\min }-1\right)\right)
\end{aligned}
$$

Using the above notations, the conditions for the existence of a solution, calculations, and all related considerations are the same as those for Section 4.2. The solution is given in the final row of Table 6 .

\begin{tabular}{|c|c|}
\hline $\begin{array}{l}\text { existence } \\
\text { condition }\end{array}$ & $e_{1}^{\min } \leq \min \left(1, \frac{\delta_{1}}{\delta_{2}-1}\left(e_{2}^{\min }-1\right)\right)$ \\
\hline $\begin{array}{l}\text { extremes of } \\
\text { the feasible } \\
\text { P.O. } \\
\text { boundary }\end{array}$ & $\begin{array}{l}\mathrm{L}=\left(L_{1}, L_{2}\right)=\left(e_{1}^{\min }, \frac{\delta_{2}-1}{\delta_{1}} e_{1}^{\min }+1\right) \\
\mathrm{R}=\left(\frac{\delta_{1}}{\delta_{2}-1}\left(\max \left(\frac{\delta_{2}-1}{\delta_{1}}+1, e_{2}^{\min }\right)-1\right), \max \left(\frac{\delta_{2}-1}{\delta_{1}}+1, e_{2}^{\min }\right)\right)\end{array}$ \\
\hline $\begin{array}{l}\text { optimal } \\
\text { effects }\end{array}$ & $\begin{array}{l}x^{*}=\left(w_{1}, w_{2}\right) \\
w_{1}=\delta_{1} /\left(r \delta_{1}-\delta+1\right) \\
w_{2}=r w_{1}\end{array}$ \\
\hline
\end{tabular}

Table 6. The optimal solution in type $2 T$ 
Table 6. cont.

\begin{tabular}{|c|c|c|c|}
\hline & \multicolumn{2}{|l|}{$r>L_{2} / L_{1}$} & $x^{*}=L$ \\
\hline & \multicolumn{2}{|l|}{$r<R_{2} / R_{1}$} & $x^{*}=R$ \\
\hline \multirow[t]{4}{*}{$\begin{array}{l}\text { optimal } \\
\text { solution }\end{array}$} & \multicolumn{2}{|c|}{$\begin{array}{l}L_{2} / L_{1} \leq r \leq \\
R_{2} / R_{1}\end{array}$} & $\begin{array}{l}q_{1}^{*}=1 /\left(r \delta_{1}-\delta_{2}+1\right) \\
q_{2}^{*}=1\end{array}$ \\
\hline & \multicolumn{2}{|l|}{$r>L_{2} / L_{1}$} & $\begin{array}{l}q_{1}^{*}=e_{1}^{\min } / \delta_{1} \\
q_{2}^{*}=1\end{array}$ \\
\hline & \multirow[t]{2}{*}{$r<R_{2} / R_{1}$} & $\delta_{1}=1$ & $\begin{array}{l}q_{1}^{*}=R_{1} \\
q_{2}^{*}=1\end{array}$ \\
\hline & & $\begin{array}{ll}\delta_{1} & > \\
1\end{array}$ & $\begin{array}{l}q_{1}^{*}=\frac{\frac{\delta_{1}}{\delta_{2}-1} \max \left(\frac{\delta_{2}-1}{\delta_{1}}+1, e_{2}^{\min }\right)-1}{1+q_{2}\left(\delta_{1}-1\right)} \\
q_{2}^{*}=\frac{\left(\delta_{1}-\vartheta-1\right)+\sqrt{\left(\delta_{1}-\vartheta-1\right)^{2}+4 \vartheta\left(\delta_{1}-1\right)}}{2\left(\delta_{1}-1\right)} \\
\vartheta=\max \left(\frac{\delta_{2}-1}{\delta_{1}}+1, e_{2}^{\min }\right)\end{array}$ \\
\hline
\end{tabular}

\subsection{TYPES 3 AND 4 TRUNCATED}

Types 3 and 4 truncated are the same as those of the bilinear free case. We therefore refer the reader to the considerations given in Sections 4.3 and 4.4 .

\section{AN ALGORITHM}

The input data is $\delta, e^{\mathrm{min}}$, and the option free-truncated function.

We begin by acquiring the data and by doublechecking the conditions required in Section 2.

With regard to $r$, it is quite possible that the user is unable to determine this a priori, and it is therefore useful to supply the user with an interval of variability $r_{-}$int to allow this parameter to be established.

The algorithm proceeds using the tables given in Sections 4 and 5 . If a feasible solution is reached, the process stops. Otherwise, the user has to be informed that $e_{1}{ }^{\text {min }}$ and/or $e_{2}{ }^{\min }$ are too binding and should be modified, giving suitable indications for doing this.

A definitive calculation can now be made and the results communicated.

\section{SOME APPLICATIONS}

In Industrial Economics, finding the optimal quantities of goods to be produced is a well-known problem. Some goods may be complementary or substitutes; hence, their demands may influence each other. If the same firm produces such kinds of goods, 
it is profitable to optimally decide the production quantities of each product. This decision also depends on the willingness of the decision-maker to potentially sacrifice part of the demand of one product. This willingness to cannibalize a product depends on various factors, examples being the future market situation of the two products and a company's desire to place itself at a strategic advantage in an emerging market (for a detailed analysis of the factors influencing the willingness to cannibalize, see Chandy et al., 1998; Nijssen et al., 2004 and Battaggion et al., 2009).

The model can be used analogously in Public Economics to calibrate two differing economic policies that are interfering with each other.

In Medicine and Veterinarian practice, the balance of interfering drugs is usually performed by successive approximations, keeping the patient monitored.

Finally, further applications can be seen in Zootechnics (to optimize diets), in Agriculture (to calculate dosages of parasiticides or additives so as to increase production), and so on.

\section{SOME OPEN PROBLEMS}

Figure 8 shows a graph corresponding to Figure 1 for the case $n=3$. Working with graphic methods (as in this paper) is more difficult in the case of multilinear functions, but not impossible.
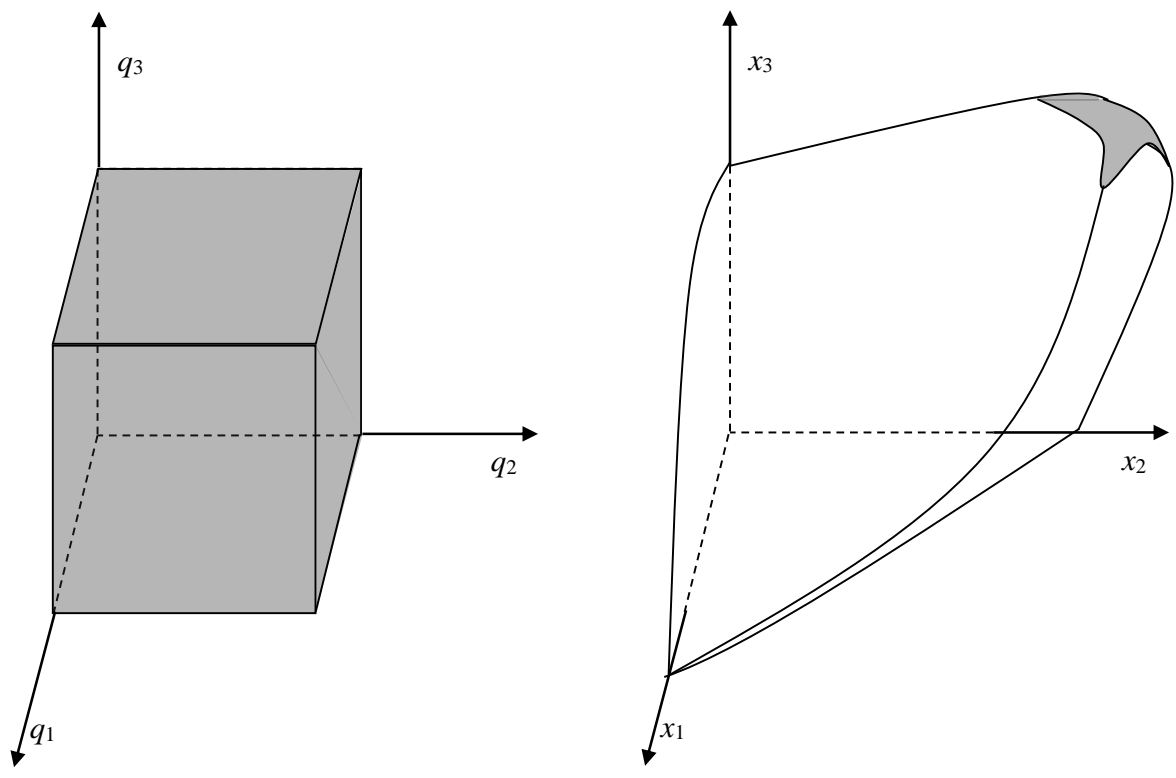

Fig. 8. $n=3$

Further studies could apply this technique to Cooperative Game Theory, where bilinear functions are often applied (see Fragnelli and Gambarelli, 2013a, 2013b). 


\section{ACKNOWLEDGMENTS}

This work is sponsored by Research Grants of University of Bergamo. The authors warmly thank Angelo Uristani for his fundamental help in the preparatory phase.

\section{REFERENCES}

Agreste, S., Carfi, D., Ricciardello, A., 2012. An algorithm for payoff space in $\mathrm{C}^{1}$ parametric games. APPS /Applied Sciences, Balkan Society of Geometers, 14, pp. 1-14. http://www.mathem.pub.ro/apps/v14/A14-ag.pdf.

Arthanari, T., Carfi, D., Musolino, F., 2015. Game Theoretic Modeling of Horizontal Supply Chain Coopetition among Growers. International Game Theory Review, World Scientific, 17(2). https://dx.doi.org/10.1142/S0219198915400137.

Baglieri, D., Carfi, D., Dagnino, G.B., 2012. Asymmetric R\&D Alliances and Coopetitive Games. In: Advances in Computational Intelligence: $14^{\text {th }}$ International Conference on Information Processing and Management of Uncertainty in Knowledge-Based Systems, IPMU 2012, Catania, Italy, July 2012, Proceedings, Part IV, ed. by S. Greco, B. BouchonMeunier, G. Coletti, M. Fedrizzi, B. Matarazzo, and R.R. Yager. Springer-Verlag, Berlin Heidelberg, pp. 607-621. https://dx.doi.org/10.1007/978-3-642-31724-8_64.

Battaggion M.R., Grieco D., 2009. R\&D Competition with Radical and Incremental Innovation. Rivista Italiana degli Economisti, 2.

Carfi, D., 2008. Optimal Boundaries for Decisions. AAPP /Physical, Mathematical, and Natural Sciences. 86(1), pp. 1-11. https://dx.doi.org/10.1478/C1A0801002.

Carfi, D., 2009a. Complete study of linear infinite games. In: Proceedings of the International Geometry Center - Prooceding of the International Conference "Geometry in Odessa 2009", Odessa, May 25-30, 2009, 2(3), pp. 19-30. http://d-omega.org/category/ books-and-papers/.

Carfi, D., 2009b. Decision-Form Games. In: Communications to SIMAI Congress - Proceedings of the $9^{\text {th }}$ Congress of SIMAI, the Italian Society of Industrial and Applied Mathematics, Roma (Italy. September 15-19, 2008, Proceedings, ed. by E. De Bernardis, G. Fotia, and L. Puccio, 3(307), pp. 1-12. https://dx.doi.org/10.1685/CSC09307.

Carfi, D., 2009c. Differentiable Game Complete Analysis for Tourism Firm Decisions. In: Proceedings of the 2009 International Conference on Tourism and Workshop on Sustainable Tourism within High Risk Areas of Environmental Crisis, pp. 1-10. Also available as MPRA Paper 29193 at http://mpra.ub.uni-muenchen.de/29193/.

Carfi, D., 2009d. Globalization and Differentiable General Sum Games. In: Proceedings of the $3^{\text {rd }}$ International Symposium "Globalization and convergence in economic thought", Bucharest, December 11-12, 2009, Bucaresti, Editura ASE.

Carfi, D., 2009e. Payoff space in $\mathrm{C}^{1}$-games. Applied Sciences (APPS), Balkan Society of Geometers, 11, pp. 35-47. http://www.mathem.pub.ro/apps/v11/A11-ca.pdf

Carfi, D., 2010. A model for Coopetitive Games. MPRA Paper 59633. http://mpra.ub.uni-muenchen.de/59633/

Carfi, D., 2012a. Coopetitive Games and Applications. In: R.K. Mishra, S. Deman, M. Salunkhe, S.B. Rao, and J. Raveendran (eds), Advances and Applications in Game Theory, J. Macmillan, India, pp. 128-147. Only printed version.

Carfi, D., 2012b. Payoff space for $\mathrm{C}^{1}$ games. In: D. Carfi, A. Ricciardello, APPS /Applied Sciences, Monographs 9, Geometry Balkan Press, Bucharest, pp. 74-89. http://www. mathem.pub.ro/apps/mono/A-09-Car.pdf. 
Carfi, D., Fici, C., 2012. The government-taxpayer game. In: L. Ungureanu (ed.), Theoretical and Practical Research in Economic Fields, ASERS, 3, 1(5). Summer 2012, pp. 13-26. http://www . asers.eu/journals/tpref/tpref-past-issues.html.

Carfi, D., Gambarelli, G. and Uristani, A., 2013. Balancing pairs of interfering elements. In: Zarzadzanie finansami. Mierzenie wyników przedsiębiorstw $i$ ocena efektywności inwestycji [Financial management, measurement of performance and evaluation of investment appraisal], Zeszyty Naukowe Uniwersytetu Szczecińskiego, Nr 760, Finanse, Rynki Finansowe, Ubezpieczenia Nr 59, Szczecin University Press, Szczecin, pp. 435-442. http://www.researchgate.net/publication/259323035_Balancing_pairs_of_ interfering_elements

Carfi, D., Lanzafame, F., 2013. A Quantitative Model of Speculative Attack: Game Complete Analysis and Possible Normative Defenses. In: M. Bahmani-Oskooee and S. Bahmani (eds), Financial Markets: Recent Developments, Emerging Practices and Future Prospects. Nova Science Publishers, pp. 173-194.

https://www.novapublishers.com/catalog/product_info.php?products_id=46483.

Carfi, D., Musolino F., 2011a. Fair Redistribution in Financial Markets: a Game Theory Complete Analysis. Journal of Advanced Studies in Finance, ASERS Publishing House, 2, 2(4), pp. 74-100. http://asers.eu/journals/jasf/jasf-past-issues.html.

Carfi, D., Musolino, F., 2011b. Game Complete Analysis for Financial Markets Stabilization. In: Proceedings of the $1^{\text {st }}$ International On-line Conference on Global Trends in Finance, pp. 14-42, ASERS. http://www.asers.eu/asers_files/conferences/GTF/GTF_ eProceedings_last.pdf.

Carfi, D., Musolino, F., 2012a. A coopetitive approach to financial markets stabilization and risk management. In: Advances in Computational Intelligence, Part IV. $14^{\text {th }}$ International Conference on Information Processing and Management of Uncertainty in KnowledgeBased Systems, IPMU 2012, Catania, Italy, July 9-13, 2012, Proceedings, Part IV, ed. by S. Greco, B. Bouchon-Meunier, G. Coletti, M. Fedrizzi, B. Matarazzo, and R. Yager, Springer, Berlin-Heidelberg, serie Communication in Computer and Information Science, 300, pp. 578-592. https://dx.doi.org/10.1007/978-3-642-31724-8_62.

Carfi, D., Musolino, F., 2012b. Game Theory and Speculation on Government Bonds. Economic Modelling, Elsevier, 29(6), pp. 2417-2426.

https://dx.doi.org/10.1016/j.econmod.2012.06.037.

Carfi, D., Musolino, F., 2012c. Game Theory Models for Derivative Contracts: Financial Markets Stabilization and Credit Crunch, Complete Analysis and Coopetitive Solution. Lambert Academic Publishing. https://www.lap-publishing.com/ catalog/details//store/gb/book/978-3-659-13050-2/game-theory-models-forderivative-contracts.

Carfi, D., Musolino, F., 2013a. Credit Crunch in the Euro Area: A Coopetitive Multiagent Solution. In: A.G.S. Ventre, A. Maturo, S. Hoskova-Mayerova, J. Kacprzyk (eds), Multicriteria and Multiagent Decision Making with Applications to Economic and Social Sciences: Studies in Fuzziness and Soft Computing, Springer Verlag, Berlin Heidelberg, 305, pp. 27-48, https://dx.doi.org/10.1007/978-3-642-35635-3\_3.

Carfi, D., Musolino, F., 2013b. Game Theory Appication of Monti's Proposal for European Government Bonds Stabilization. In: V. Balan (ed.), APPS /Applied Sciences, Balkan Society of Geometers, 15, pp. 43-70. http://www.mathem.pub.ro/apps/v15/A15-ca. pdf.

Carfi, D., Musolino, F., 2013c. Model of Possible Cooperation in Financial Markets in Presence of Tax on Speculative Transactions. AAPP/Physical, Mathematical, and Natural Sciences, 91(1), pp. 1-26. https://dx.doi.org/10.1478/AAPP.911A3. 
Carfi, D., Musolino, F., 2014a. Dynamical Stabilization of Currency Market with Fractallike Trajectories. Scientific Bulletin of the Politehnica University of Bucharest, Series A-Applied Mathematics and Physics, 76(4), pp. 115-126. http://www. scientificbulletin.upb.ro/rev_docs_arhiva/rezc3a_239636.pdf.

Carfi, D., Musolino, F., 2014b. Speculative and Hedging Interaction Model in Oil and U.S. Dollar Markets with Financial Transaction Taxes. Economic Modelling, 37, pp. 306-319. https://dx.doi.org/10.1016/j.econmod.2013.11.003.

Carfi, D., Musolino, F., 2015a. A Coopetitive-Dynamical Game Model for Currency Markets Stabilization. AAPP /Physical, Mathematical, and Natural Sciences, 93(1), pp. 1-29. https://dx.doi.org/10.1478/AAPP.931C1.

Carfi, D., Musolino, F., 2015b. Tax Evasion: a Game Countermeasure. AAPP /Physical, Mathematical, and Natural Sciences, 93(1), pp. 1-17. https://dx.doi.org/10.1478/ AAPP. 931C2.

Carfi, D., Musolino, F., Ricciardello, A., Schilirò, D., 2012. Preface: Introducing PISRS. AAPP /Physical, Mathematical, and Natural Sciences, 90 Supplement No. 1, PISRS Proceedings (Part I), ed. by D. Carfì, F. Musolino, A. Ricciardello, and D. Schilirò, pp. 1-4. https://dx.doi.org/10.1478/AAPP.90S1E1.

Carfi, D., Musolino, F., Schilirò, D., Strati, F., 2013. Preface: Introducing PISRS (Part II). AAPP /Physical, Mathematical, and Natural Sciences, 91, Supplement No. 2, PISRS Proceedings (Part II), ed. by D. Carfì, F. Musolino, D. Schilirò, and F. Strati. https: //dx.doi.org/10.1478/AAPP.91S2E1.

Carfi, D., Patane, G., Pellegrino, S., 2011. Coopetitive Games and Sustainability in Project Financing. In: G. Calabrò, A. D’Amico, M. Lanfranchi, G. Moschella, L. Pulejo, R. Salomone, Moving from the Crisis to Sustainability: Emerging Issues in the International Context, Franco Angeli, pp. 175-182 http://www.francoangeli.it/Ricerca/Scheda_ libro. aspx?CodiceLibro $=365.906$.

Carfi, D., Perrone, E., 2011a. Asymmetric Bertrand Duopoly: Game Complete Analysis by Algebra System Maxima. Mathematical Models in Economics, ASERS Publishing House, pp. 44-66. http://www.asers.eu/asers-publishing/collections.html. Also available as MPRA Paper 35417 at http://mpra.ub.uni-muenchen.de/35417/.

Carfi, D, Perrone, E., 2011b. Game Complete Analysis of Bertrand Duopoly. Theoretical and Practical Research in Economic Fields, ASERS Publishing House, 2, 1(3), pp. 5-22. http://www . asers.eu/journals/tpref/tpref-past-issues.html.

Carfi, D., Perrone, E., 2011c. Game Complete Analysis of Bertrand Duopoly. Mathematical Models in Economics, ASERS Publishing House, pp. 22-43. http://www.asers.eu/ asers-publishing/collections.html.

Carfi, D., Perrone, E., 2012a. Game complete analysis of symmetric Cournout duopoly. MPRA Paper 35930, University Library of Munich, Germany. http://mpra.ub.uni-muenchen. de/35930/.

Carfi, D., Perrone, E., 2012b. Game Complete Analysis of Classic Economic Duopolies. Lambert Academic Publishing. https://www.lap-publishing.com/catalog/details/ /store/ru/book/978-3-8484-2099-5/game-complete-analysis-of-classiceconomic-duopolies.

Carfi, D., Perrone E., 2013. Asymmetric Cournot Duopoly: A Game Complete Analysis. Journal of Reviews on Global Economics, 2, pp. 194-202. https://dx.doi.org/10.6000/ 1929-7092.2013.02.16.

Carfi, D., Pintaudi, A., 2012. Optimal Participation in Illegitimate Market Activities: Complete Analysis of 2 Dimimensional Cases. Journal of Advanced Research in Law and Economics, ASERS, 3 1(5), pp. 10-25. http://www. asers.eu/journals/jarle/jarlepast-issues.html. 
Carfi, D., Ricciardello, A., 2009. Non-reactive strategies in decision-form games. AAPP /Physical, Mathematical, and Natural Sciences, 87(2), pp. 1-12. https://dx.doi.org/ 10.1478/C1A0902002.

Carfi, D., Ricciardello, A., 2010. An algorithm for payoff space in $\mathrm{C}^{1}$ Games. AAPP /Physical, Mathematical, and Natural Sciences, 88(1), pp. 1-19. https://dx.doi.org/10.1478/ C1A1001003.

Carfi, D., Ricciardello A., 2012a. Algorithms for Payoff Trajectories in $\mathrm{C}^{1}$ Parametric Games. In: Advances in Computational Intelligence: $14^{\text {th }}$ International Conference on Information Processing and Management of Uncertainty in Knowledge-Based Systems, IPMU 2012, Catania, Italy, July 2012, Proceedings, Part IV, ed. by S. Greco, B. BouchonMeunier, G. Coletti, M. Fedrizzi, B. Matarazzo, and R.R. Yager, Springer-Verlag, Berlin Heidelberg, pp. 642-654. https://dx.doi.org/10.1007/978-3-642-31724-8\_67.

Carfi, D., Ricciardello, A., 2012b. Topics in Game Theory. Applied Sciences - Monographs No. 9, Balkan Society of Geometers. Balkan Society of Geometers. http://www.mathem. pub.ro/apps/mono/A-09-Car.pdf.

Carfi, D., Ricciardello, A., 2013a. An Algorithm for Dynamical Games with Fractal-Like Trajectories. Fractal Geometry and Dynamical Systems in Pure and Applied Mathematics II: Fractals in Applied Mathematics. PISRS 2011 International Conference on Analysis, Fractal Geometry, Dynamical Systems and Economics, Messina, Italy, November 8-12, 2011 - AMS Special Session on Fractal Geometry in Pure and Applied Mathematics: in memory of Benoit Mandelbrot, Boston, Massachusetts, January 4-7, 2012 - AMS Special Session on Geometry and Analysis on Fractal Spaces, Honolulu, Hawaii, March 3-4, 2012. 601: 95-112 of Contemporary Mathematics. American Mathematical Society. Ed. by D. Carfi, M. Lapidus, E. Pearse, and M. Van Frankenhuijsen. https://dx.doi.org/10.1090/conm/601/11961.

Carfi, D., Ricciardello, A., 2013b. Computational representation of payoff scenarios in $\mathrm{C}^{1}$ families of normal-form games. Uzbek Mathematical Journal, 1, pp. 38-52. Only printed version.

Carfi, D., Romeo, A., 2015. Improving Welfare in Congo: Italian National Hydrocarbons Authority Strategies and its Possible Coopetitive Alliances with Green Energy Producers. Journal of Applied Economic Sciences, 10, 4(34), pp. 571-592. http://cesmaa.eu/ journals/jaes/files/JAES_summer $\ \% 204$ (34)_online.pdf

Carfi, D., Schilirò, D., 2011a. Coopetitive Games and Global Green Economy. In: G. Calabrò, A. D’Amico, M. Lanfranchi, G. Moschella, L. Pulejo, R. Salomone, Moving from the Crisis to Sustainability: Emerging Issues in the International Context, Franco Angeli, pp. 357-366. http://www.francoangeli.it/Ricerca/Scheda_libro. aspx?CodiceLibro $=365.906$.

Carfi, D., Schilirò, D., 2011b. Crisis in the Euro Area: Coopetitive Game Solutions as New Policy Tools. Theoretical and Practical Research in Economic Fields, 2, 1(3), pp. 23-36. http://www.asers.eu/journals/tpref/tpref-past-issues.html.

Carfi, D., Schilirò, D., 2011c. Crisis in the Euro Area: Co-opetitive Game Solutions as New Policy Tools. In: L. Ungureanu, Mathematical Models in Economics, ASERS, pp. 67-86. http://www . asers.eu/asers-publishing/collections.html.

Carfi, D., Schilirò, D., 2012a. A coopetitive Model for the Green Economy. Economic Modelling, Elsevier, 29(4), pp. 1215-1219. https://dx.doi.org/10.1016/j.econmod. 2012.04 .005$.

Carfi, D., Schilirò, D., 2012b. A Framework of coopetitive games: Applications to the Greek crisis. AAPP /Physical, Mathematical, and Natural Sciences, 90(1), pp. 1-32. https://dx.doi.org/10.1478/AAPP.901A1. 
Carfi, D., Schilirò, D., 2012c. A Model of Coopetitive Game for the Environmental Sustainability of a Global Green Economy. Journal of Environmental Management and Tourism, ASERS Publishing House, 3, 1(5), pp. 5-17. http://www.asers.eu/journals/ jemt/jemt-past-issues.html.

Carfì, D., Schilirò, D., 2012d. Global Green Economy and Environmental Sustainability: A Coopetitive Model. In: S. Greco, B. Bouchon-Meunier, G. Coletti, M. Fedrizzi, B. Matarazzo, and R. Yager (eds), Advances in Computational Intelligence, Part IV, $14^{\text {th }}$ International Conference on Information Processing and Management of Uncertainty in Knowledge-Based Systems, IPMU 2012, Catania, Italy, July 9-13, 2012, Proceedings, Part IV, Springer Berlin Heidelberg, serie Communication in Computer and Information Science, 300, pp. 593-606. https://dx.doi.org/10.1007/978-3-642-31724-8_63.

Carfi, D., Schilirò, D., 2013. A Model of Coopetitive Games and the Greek Crisis. In: Contributions to Game Theory and Management, Vol. VI. The Sixth International Conference Game Theory and Management, June 27-29, 2012, St. Petersburg, Russia, collected papers edited by L.A. Petrosyan and N.A. Zenkevich, Saint Petersburg State University, pp. 35-62. http://www.gsom.spbu.ru/files/upload/gtm/sbornik2012_27_05_ 2013.pdf.

Carfi, D., Schilirò, D., 2014a. Coopetitive Game Solutions for the Greek Crisis. In: D. Schilirò (ed.), Design a Pattern of Sustainable Growth, Innovation, Education, Energy and Environment, ASERS. http://www.asers.eu/asers-publishing/books.html.

Carfi, D., Schilirò, D., 2014b. Improving Competitiveness and Trade Balance of Greek Economy: A Coopetitive Strategy Model, Journal of Applied Economic Sciences, 9, 2(28), pp. 211-220. http://www.ceeol.com/aspx/issuedetails.aspx?issueid= 583d6083-2bbf-4d8c-af 1f-3b5786c6e087 \\&articleId=8c9be4cb-86d9-43f1-b555$58 \mathrm{~b} 8 \mathrm{cb} 28 \mathrm{bbeb}$.

Carfi, D., Trunfio, A., 2011. A Non-linear Coopetitive Game for Global Green Economy. In: G. Calabrò, A. D'Amico, M. Lanfranchi, G. Moschella, L. Pulejo, R. Salomone, Moving from the Crisis to Sustainability: Emerging Issues in the International Context, Franco Angeli, pp. 421-428.

http://www . francoangeli.it/Ricerca/Scheda_libro. aspx?CodiceLibro=365.906.

Chandy R.K., Tellis G.J., 1998. Organizing for Radical Product Innovation: The Overlooked Role of Willingness to Cannibalize. Journal of Marketing Research, Vol. XXXV, pp. $474-487$.

Fragnelli V., Gambarelli, G. (eds), 2013a. Open Problems in the Theory of Cooperative Games, Special Issue of International Game Theory Review, 15(2).

Fragnelli V., Gambarelli, G. (eds), 2013b. Open Problems in the Applications of Cooperative Games, Special Issue of International Game Theory Review, 15(3).

Gambarelli, G., 1990. A new approach for evaluating the Shapley value. Optimization, 21(3), pp. $445-452$.

Musolino, F., 2012. Game theory for speculative derivatives: a possible stabilizing regulatory model. AAPP /Physical, Mathematical, and Natural Sciences, 90, Supplement No.1, PISRS Proceedings (Part I), pp. 1-19. https://dx.doi.org/10.1478/AAPP.90S1C1.

Nijssen E.J., Hillebrand B., Vermeulen P.A.M., Kemp R., 2004. Understanding the Role of Willingness to Cannibalize in New Service Development. Scales Research Reports H200308, EIM Business and Policy Research.

Okura, M., Carfi, D., 2014. Coopetition and Game Theory, Journal of Applied Economic Sciences, 9, 3(29), pp. 457-468. http://cesmaa.eu/journals/jaes/files/JAES_2014_ Fall.pdf $\backslash$ \#page=123. 